\title{
Seismic attributes and integrated prediction of fractured and caved carbonate reservoirs in the Tarim Basin, China
}

\author{
Liu Lifeng', Sam Zandong Sun ${ }^{1 *}$, Yang Haijun², Han Jianfa², Gao Hongliang², \\ Jing Bing ${ }^{2}$ and Zhu Xinghui ${ }^{1}$
}

${ }^{1}$ Laboratory for Integration of Geology \& Geophysics, China University of Petroleum, Beijing 102249, China

${ }^{2}$ Research Institute of Exploration \& Development, PetroChina Tarim Oilfield Company, Korla 841000, China

(C) China University of Petroleum (Beijing) and Springer-Verlag Berlin Heidelberg 2011

\begin{abstract}
The carbonate reservoirs in the Tarim Basin are characterized by anisotropy and strong heterogeneity. Combined with an integrated analysis of data from seismic, geology, and drilling results, a series of attributes which are suitable for fractured and caved carbonate reservoir prediction is discussed, including amplitude, coherence analysis, spectra decomposition, seismic absorption attenuation analysis and impedance inversion. Moreover, 3-D optimization of these attributes is achieved by integration of multivariate discriminant analysis and principle component analysis, where the logging data are taken as training samples. Using the optimized results, the spatial distribution and configuration features of the caved reservoirs can be characterized in detail. This technique not only improves the understanding of the spatial distribution of current reservoirs but also provides a significant basis for the discovery and production of carbonate reservoirs in the Tarim Basin.
\end{abstract}

Key words: Coherence analysis, spectra decomposition, seismic absorption and attenuation, impedance inversion, seismic attribute optimization

\section{Introduction}

The Ordovician carbonate reservoirs in the Tarim Basin have been proved to be a major target for large-scale oilfields. So far large oilfields have been found one after another including the Tahe, Lunnan and Tazhong areas in the Tarim Basin. However, finding an effective prediction method for the Ordovician carbonate caves, dissolution pores and fracture zones remains a major problem to extend the exploration and reduce the drilling risk.

\section{The general characteristics of fractured and caved carbonate reservoirs}

Due to low matrix porosity and low permeability, the secondary dissolved caves and fractures become the effective storage spaces of the Ordovician carbonate rocks. The key to a high and stable production well is to drill the zone where dissolved caves and fractures are highly developed (Wang et al, 2005; Han et al, 2006; Wang et al, 2010; Pang et al, 2010).

The Tazhong area experienced multi-stage tectonic movement and crust uplifting. As a result, Ordovician carbonate formations suffered multi-stage weathering and

* Corresponding author. email: samzdsun@yahoo.com

Received March 18, 2011 dissolution, forming many unconformities accompanied with a large number of faults and fractures.

Fractures created by structural movement have important influence on the petrophysical properties of the reservoir. These fractures not only provide the storage space, but also connect pores, improving the permeability of reservoir. Meanwhile, these fractures provide channels for acid fluid such as organic acids and groundwater in the burial period, which produce and enlarge dissolution caves. Seismic detection and prediction of the secondary storage space of reservoirs can be difficult, and it is the major topic of this work.

Due to the short-term stretch effect in the late Early Permian Hercynian, volcanic eruptions and lava intrusion occurred in the Tazhong (TZ) area. Especially in the western part of the region, the large-scale distributed volcanic rocks and sub-volcanic intrusion made the fractures and caves which were closed in earlier time open again and also created new fractures and caves. When the deep hydrothermal fluids poured into the Upper Ordovician limestone formation through fractures, strong dissolution and metasomatism (such as fluorite deposition and silicification) occurred along cracks, subsequently a large number of dissolved fractures and caves were formed which greatly improved the performance of carbonate reservoirs (Fig. 1). Fig. 2 is the superposed profile of the dissolution caved reservoirs (yellow zones) predicted 


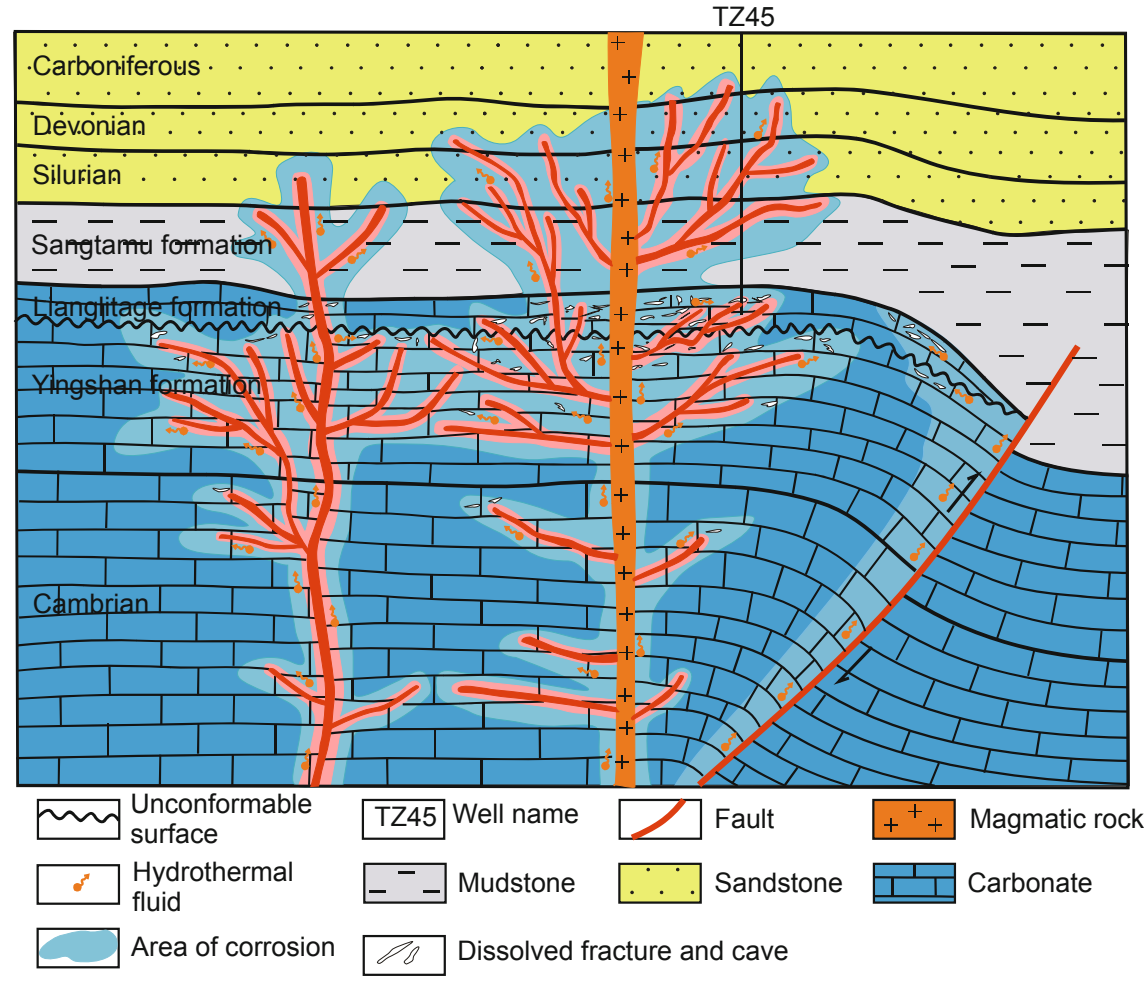

Fig. 1 The geological model chart of the fractured and caved reservoirs at the TZ45 area in the central Tarim Basin

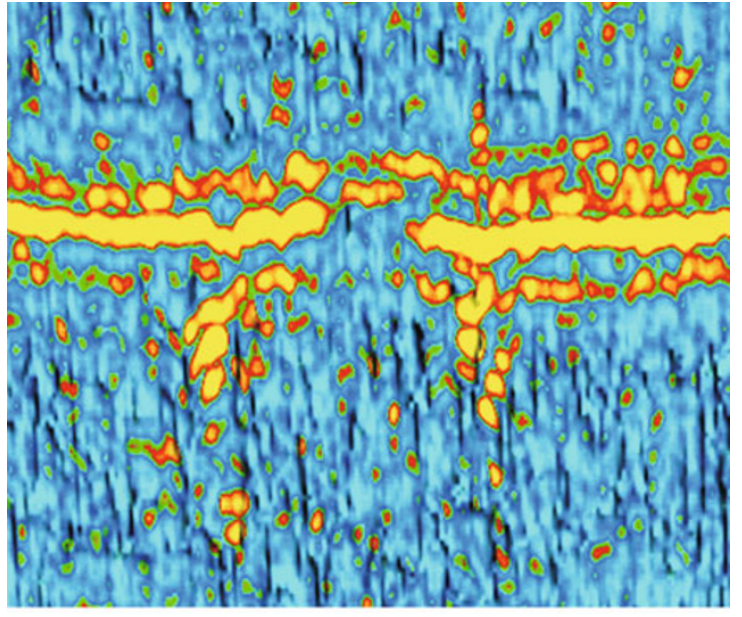

Dissolution holes
and caves

Carbonate matrix

Fig. 2 The superposed profile of frequency-division attribute and coherence attribute

by frequency-division attribute and the faults predicted by coherence attribute, showing that the dissolution caved reservoirs are developed along faults. Fluorite fillings are found in the Middle and Upper Ordovician limestone caves of TZ45, TZ12, TZ16 and TZ161 wells at the No.1 fracture zone. Especially at well TZ45, the cracks develop greatly, jointed with fluorite cleavage crack at the fluorite-rich section and formed the fracture-cave system by dissolution along the fractures.

\section{Characteristics of seismic attributes for fractured and caved carbonate reservoirs}

Seismic attributes are an important tool for reservoir prediction. By extracting physical properties, lithology and even fluid information which is hidden in seismic data, we can analyze vertical and horizontal changes of seismic information from different aspects to reveal geological anomaly phenomena and oil-gas bearing information which can not be easily found from the original seismic profiles. The TZ45 area is studied in this paper as an example for the characteristics of fractured and caved carbonate reservoirs of the Tarim Basin. Based on integrated analysis of seismic, geology and drilling data, multiple attributes are applied to the prediction of fractured and caved carbonate reservoirs and attributes which are sensitive to changes in the characteristics are selected. By optimizing these attributes, a series of effective techniques for the reservoir prediction is established, which is expected to guide reservoir prediction and exploration in the Tarim Basin.

\subsection{Amplitude at tribute}

Among all seismic attributes, the amplitude attribute is the fundamental and most important one. For carbonate reservoirs, when neglecting the factors from acquisition and processing, the amplitude is only influenced by the development of caves, holes (the size is generally smaller than one meter) but fractures. This fact could help to reveal the lateral distribution and fluid variation of reservoirs (Wen et al, 2008). The seismic reflection features of fractured and 
caved carbonate reservoirs are always as follows: the top of the holes shows relatively weaker amplitude while the amplitude at the bottom is strong, and the amplitude inside a hole is stronger than that of surrounding rocks but weaker than that of the bottom (Cai, 2005). However, the amplitude attribute cannot yield obvious results because the Ordovician carbonate is deeply buried and the available seismic signal is weak. Compared with drilling and logging data, it shows that both strong and weak amplitudes are distributed in reservoir developed areas. In areas with strong amplitude in the background of weak amplitude, fractured and caved reservoirs are more likely to develop (Fig. 3).

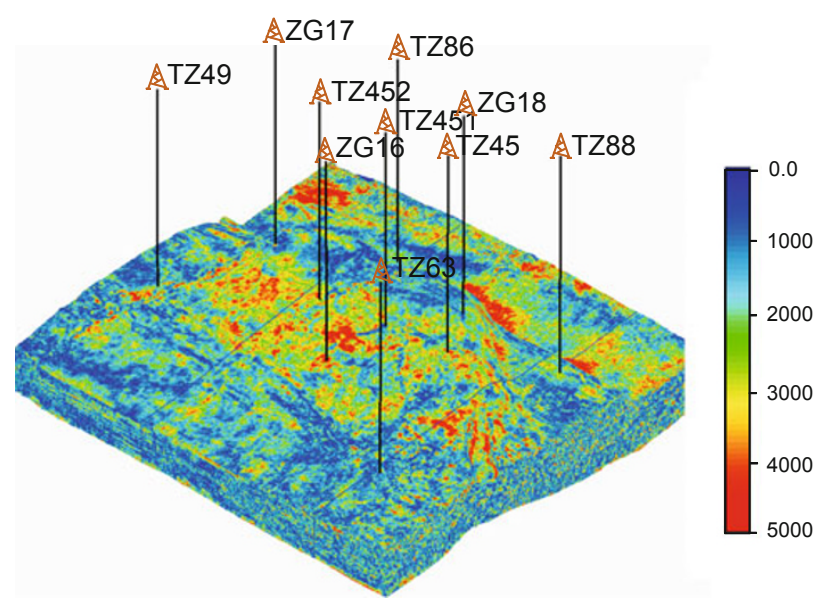

Fig. 3 The Ordovician RMS (root mean square) amplitude in TZ45 area

\subsection{Coherence cube technology}

The core of coherence cube technology is seeking the similarities while preserving differences for the seismic data in order to highlight the irrelevant information. The coherence cube data can be used to identify the horizontal nonuniformity and fracture characteristics of subtle strata (Chen et al, 2005). The fracture characteristics are clear in the TZ45 area. If the horizontal non-similarity caused by the fracture dislocation is ruled out, the factors mainly influencing the seismic relevance are fractures, dissolved caves and small cracks which form the main storage spaces of carbonate reservoirs. So the coherence cube technology can be used to predict the development zone of carbonate pores, caves and fractures. After calculating the coherence cube of the three dimensional seismic amplitude-preserved data at TZ45 area, the fracture and cave development characteristics of the carbonate reservoirs are detected using the intrinsic algorithm. High-yield wells are in the regions of weak coherent values (dark), and the wells in the strong value regions are relatively poor (Fig. 4).

However, the coherence cube predicts the development zones of the carbonate pores, caves and fractures only at a macro scale, not directly. So some uncertain factors would be inevitably confused. Besides, this method only uses the coherence of data and highlights all the discontinuous waveform situations which would confuse the understanding of anomalies resulting from geological factors. Because the horizontal strata change is assumed as a gradual process, the explanation results inevitably face the uncertainty and ambiguity in complex carbonate reservoirs. Thus other methods should be synthetically considered.

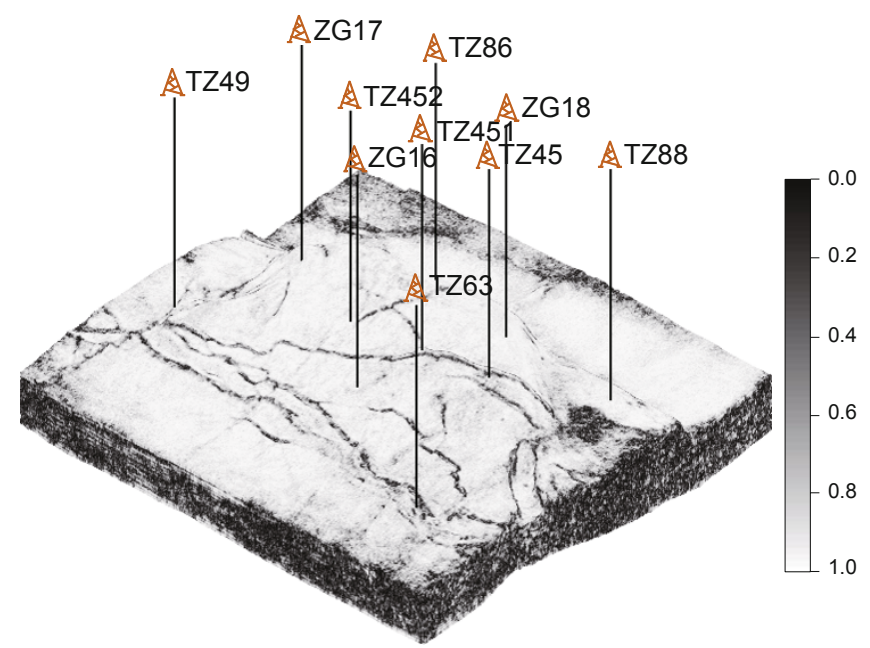

Fig. 4 The Ordovician coherence data in the Tazhong 45 area

\subsection{Spectra decomposition technology}

The spectra decomposition is implemented using the method of non-orthogonal Gabor-Morlet wavelet transform for reservoir prediction. This method uses frequency scale parameters directly to control the spectra decomposition, which avoids deficiencies between the scale parameter of the conventional wavelet transform and the frequency. Meanwhile, it overcomes the impact of the size of the time window on results in the conventional spectral decomposition method and emphasizes the local characteristics of the seismic signal. As a result, it greatly enhances the stability and resolution (Yuan, 2007).

A series of frequency division data with different frequency is extracted. Comparison with the drilling data suggests that the $22 \mathrm{~Hz}$ data is the most sensitive to the Ordovician fractured and caved reservoirs. Thus the frequency division data at $22 \mathrm{~Hz}$ are more suitable to indicate the spatial distribution of reservoirs in the area (Fig. 5).

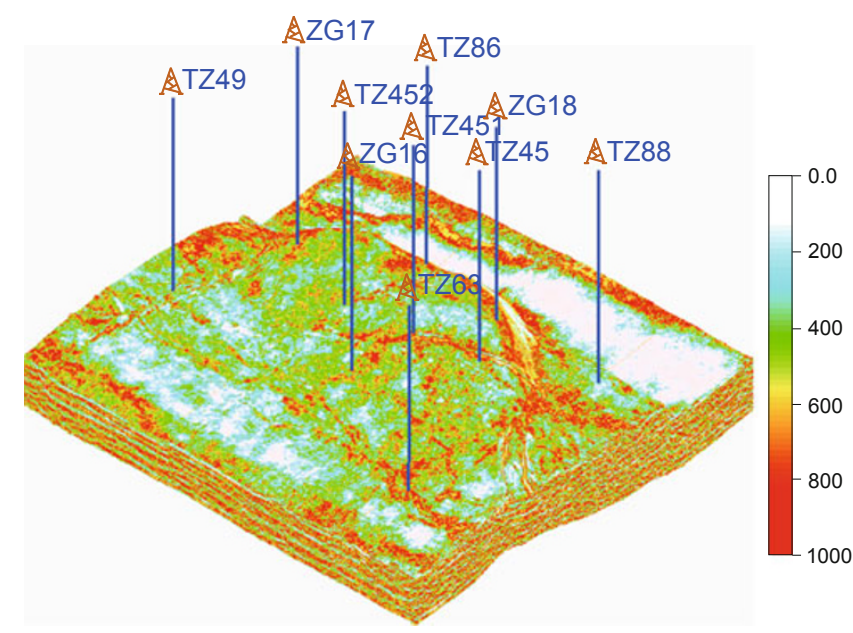

Fig. 5 The Ordovician frequency division data $(22 \mathrm{~Hz})$ in the Tazhong 45 area 


\subsection{Seismic absorption and attenuation technology}

Seismic absorption and attenuation refers to the loss of seismic wave energy during its propagation, it is the internal attribute of the medium (Huang et al, 2000). A large amount of forward modeling and theory research has proved that, when a seismic wave propagates through oil and gas reservoirs, the high frequency components are preferentially absorbed (Sams et al, 1997; Sinha et al, 2005). This is called "high frequency absorption".

The frequency attenuation gradient (FAG) is the most sensitive attribute for fluid detection and reservoir distribution prediction among the absorption attenuation attributes. FAG here means the fitted slope of high frequency amplitude based on the spectra decomposition. It indicates the variation of high-frequency seismic energy versus the frequency, which can be used to denote the speed of attenuation in the seismic propagation process ( $\mathrm{Bi}$ et al, 2007). Fig. 6 shows the results of the FAG at the Tazhong 45 area. The high FAG corresponds to the high-production wells such as the TZ45, TZ451, ZG16 and ZG17, while the low production wells are distributed at the low FAG zones.

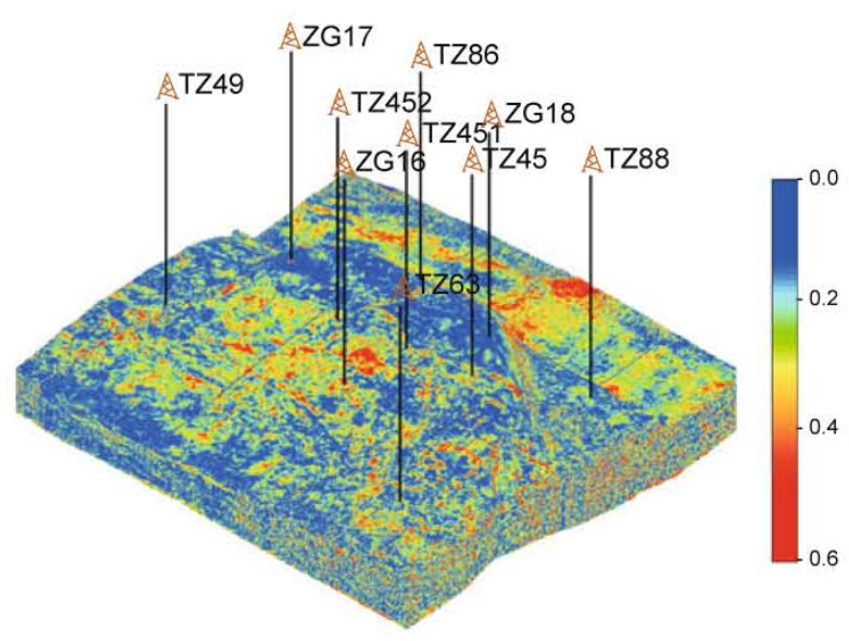

Fig. 6 The Ordovician frequency attenuation gradient data of the Tazhong 45 area

\subsection{Post-stack impedance inversion}

For the post-stack impedance inversion, the logging data are used as constraint, the forward modeling and inversion are combined and multiple-iterations are performed to obtain the impedance data (Liu et al, 1999; Jin et al, 2003).

Comparing the drilling information with the impedance inversion result, we can see that the distribution of reservoirs can be reliably predicted by impedance inversion. Generally speaking, the base rock of carbonate is usually featured as high velocity, high density, and high impedance. However, if there are cracks or erosion holes in carbonate, its velocity, density, and impedance will decrease. Therefore, considering that the reservoir is characterized by low impedance, low velocity and high porosity, the multi-well-constrained impedance inversion could be preferable to predict carbonate reservoirs.
On the profile (Fig. 7), weak impedance corresponds to large caves and fractures. Seen from Fig. 8, the weak impedance generally corresponds to the areas where caves and fractures develop. Thus, the base rock of carbonate reservoir shows high impedance, and fractures and caves are weak impedance under the background of strong impedance.

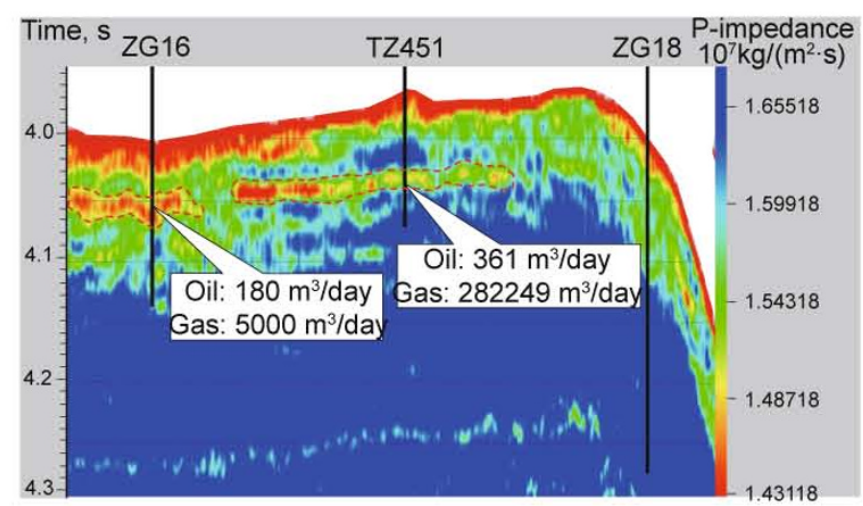

Fig. 7 The Ordovician impedance section in the Tazhong 45 area

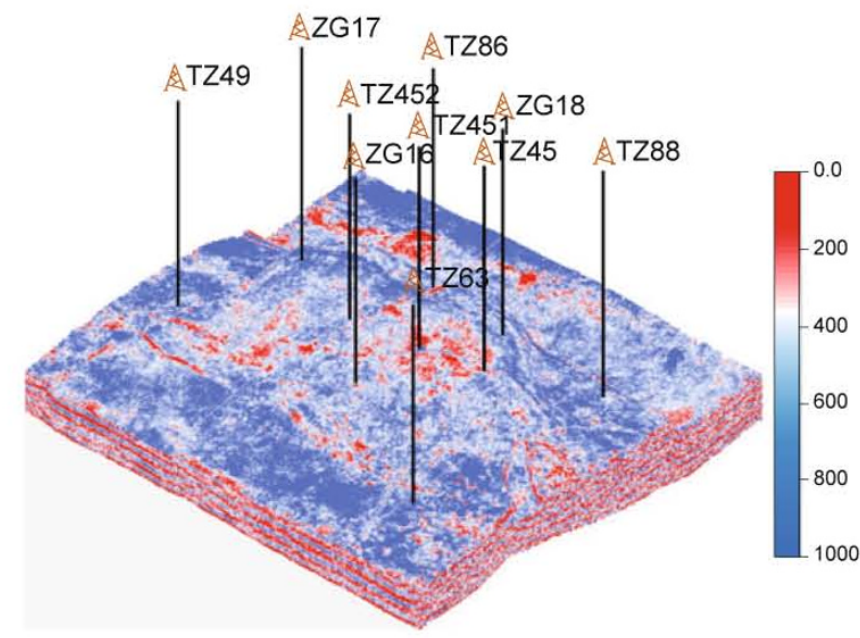

Fig. 8 The Ordovician impedance volume in the Tazhong 45 area

\section{Seismic attribute optimization and integrated prediction}

Although each kind of seismic attribute reflects some information of the reservoirs, they all have limitations and the prediction precision is not very high (Yin and Zhou, 2005). Especially for the complex carbonate reservoirs in the Tarim Basin, blind application may even cause wrong prediction results, so it is essential to set up a high performance and accurate seismic attribute optimization method that is available for carbonate reservoirs. In this paper, special attributes such as coherence cube and impedance are all put into the category of general seismic attributes, in order to be optimized with the conventional attributes and to comprehensively predict the carbonate reservoirs.

The paper introduces a 3D seismic multi-attribute optimization technique combining multiple statistical methods such as multivariate discriminant analysis, kernel 
principal component analysis and comprehensive discriminant analysis. This method has proven to be an effective approach to optimize the complicated relationships between seismic attributes and reservoir prediction, and it can obviously enhance seismic reservoir prediction accuracy, realizing the fine sculpture of carbonate dissolution cave reservoirs in $3 \mathrm{D}$ space.

The specific optimization programs are as follows:

(1) Optimization of seismic attribute. Reasonable seismic attribute subsets are chosen from seismic attribute sets, mainly considering the physical meaning, sensitivity and pertinence of the seismic attribute. These include all the seismic attributes mentioned above as well as several conventional attributes such as instantaneous frequency, arc length, energy half-time and frequency tuning energy. On the basis of that, we conduct coherence analysis between different attributes and choose the attributes sets which bear little coherence between each other. Finally, eight types of advantageous seismic attributes are determined.

(2) Seismic attribute preprocessing. Central standardization (standardization-zero) processing is employed to the optimized seismic attributes to convert values of various attributes to the same scale.

(3) Sample-training selection. According to the real reservoir condition revealed by drilling data in the study area, the potential Ordovician carbonate reservoirs are classified into hydrocarbon reservoir, hydrocarbon-showing reservoir, and non-reservoir. The three kinds are regarded as the classification basis of building a discrimination model. Reservoir types of different formations are determined by combining log data, oil-testing data, and drilling data. For this case, the reservoir types in the region of $100 \mathrm{~m}$ away from the well location are assumed the same to those in the well location, and 30,628 attribute points in the region are chosen as given training samples for attribute optimization. Based on the analysis above, the training samples include 2,536 hydrocarbon points, 3,904 hydrocarbon-showing points, and 24,188 non-reservoir points.
(4) Multivariate step-by-step discrimination. The influence of different seismic attributes on reservoir parameters is different. In this case, the contribution of eight preferred seismic attribute volumes determined above to the discriminant model is computed using multivariate step-bystep discrimination. It is found that the frequency attenuation gradient has the greatest contribution. Other attributes such as impedance, frequency tuning energy, energy half-time, rootmean-square amplitude and instantaneous frequency also have a positive contribution. Therefore the discrimination function is established on the basis of the six attributes above and a significant discrimination result can be obtained. Coherence data and arc length have the lowest contribution and even have an interference effect, so they are rejected in the step-by-step discrimination.

(5) Kernel principal component analysis. In most cases, the spatial dimension of selected seismic attribute sets is still high, bringing redundant information, which directly influences the stability of the prediction result. Therefore, kernel principal component analysis is employed to transform original seismic attribute parameters into an array of independent new variables. By choosing Gauss radial base kernel function, kernel principal component analysis is performed for the six seismic attributes introduced by multivariate step-by-step discriminant analysis. The principal component models are built and then the original attributes are transformed into independent new variables.

(6) Discriminant model building. According to the selected training samples, the discriminant analysis based on Bayesian criteria is performed for the kernel principal components (Sun, 1999). Then we reintroduce the various indexes of given training samples into the built discriminant models. Through calculating the coincidence rate of reintroducing, the discriminant ability of the model can be examined. The calculation results show that the total coincidence rate reaches $90.13 \%$, suggesting that the discriminant model is reliable and available for discriminating various reservoir types (as shown in Table 1).

Table 1 The results of discriminate analysis by using given training samples

\begin{tabular}{|c|c|c|c|c|c|}
\hline \multirow{2}{*}{ Real classification } & \multicolumn{3}{|c|}{ Prediction classification } & \multirow{2}{*}{$\begin{array}{c}\text { Coincidence rate } \\
\%\end{array}$} & \multirow{2}{*}{$\begin{array}{c}\text { Total coincidence rate } \\
\%\end{array}$} \\
\hline & Hydrocarbon layer & Hydrocarbon showing layer & Non-reservoir layer & & \\
\hline I: Hydrocarbonl ayer & 2186 & 212 & 138 & 86.20 & \\
\hline II: Hydrocarbon showing layer & 272 & 3448 & 184 & 88.32 & 90.13 \\
\hline III: Non-reservoir layer & 962 & 1254 & 21972 & 90.84 & \\
\hline
\end{tabular}

(7) Integrated prediction. We put the attribute points into the discriminant model built above, and obtain the comprehensive optimized reservoir attribute. Then, the quantitative prediction of the carbonate reservoirs can be performed. The seismic multi-attribute optimization method comprehensively considers the effective information of many seismic attributes as well as eliminating the attributes interfering with the prediction results, while the multivariate step-by-step discriminant analysis guarantees the stability of calculation results. The reliability of prediction results is also improved by effectively considering the non-linear relationships among various seismic attributes.

As shown in Fig. 9, the red zone, green zone and blue zone respectively denote hydrocarbon reservoir, hydrocarbonshowing reservoir and non-reservoir. The figure shows sections of multi-attribute optimization data volume across wells including three commercial oil/gas production wells (TZ451, ZG16, and ZG17) and one oil/gas showing well (ZG18). The red rows denote the oil/gas production segment of various wells derived from oil testing results, and yellow rows denote that derived from logging interpretation. Comparing the optimization results with oil testing or logging 
interpretation results, they are consistent with each other, meaning that the optimization result can accurately predict carbonate reservoirs and can reflect the favorable distribution range and geometric shape in $3 \mathrm{D}$ space of carbonate reservoirs.

Further, the carbonate dissolution cave reservoirs are sculpted. The obtained accurate spatial distribution of complex carbonate reservoirs underground reflects the connection relation among reservoirs, which is beneficial to complex carbonate reservoir prediction and can greatly increase the comprehensive interpretation ability and the accuracy of carbonate reservoir prediction (Fig. 10 and Fig. 11).
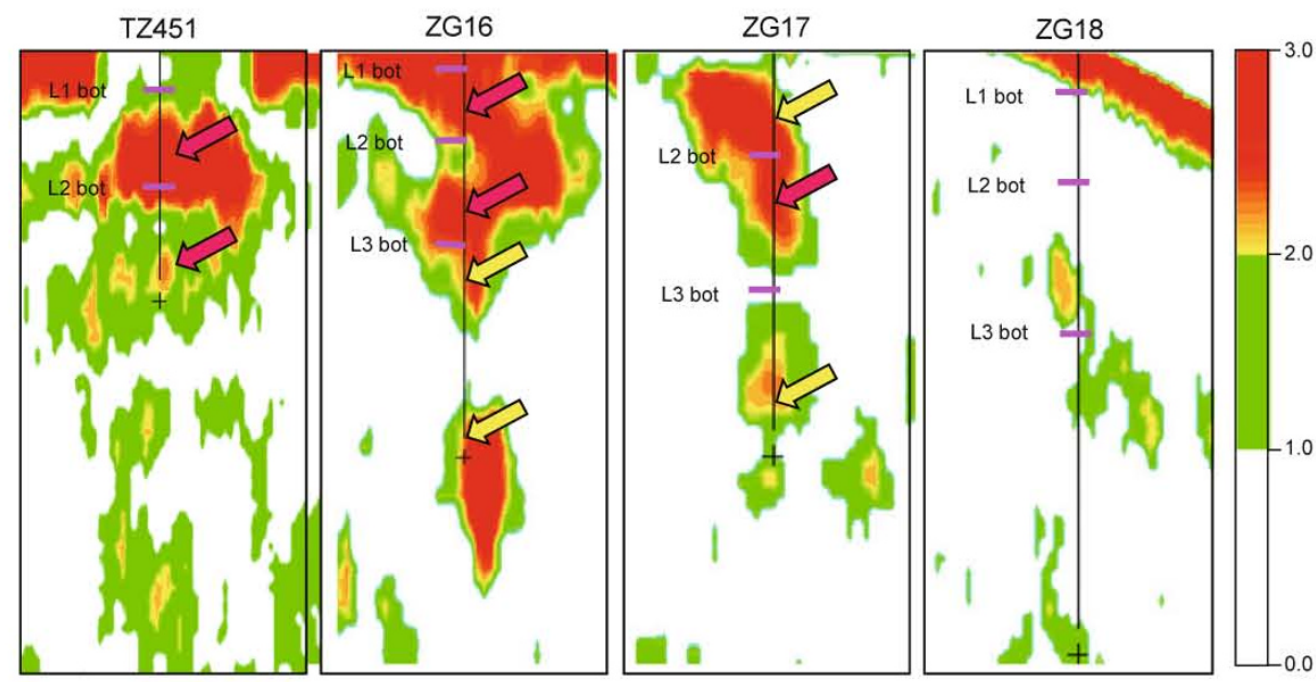

Fig. 9 Across well sections of multi-attribute optimization data volume of TZ45 area (bot: bottom; hydrocarbon layer (red); hydrocarbon showing layer (green); non-reservoir layer (white))

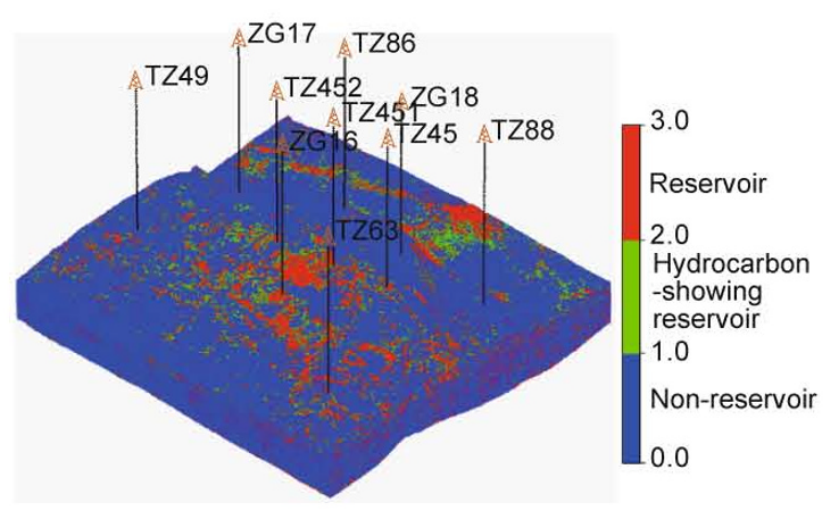

Fig. 10 The multi-attribute optimization data volume

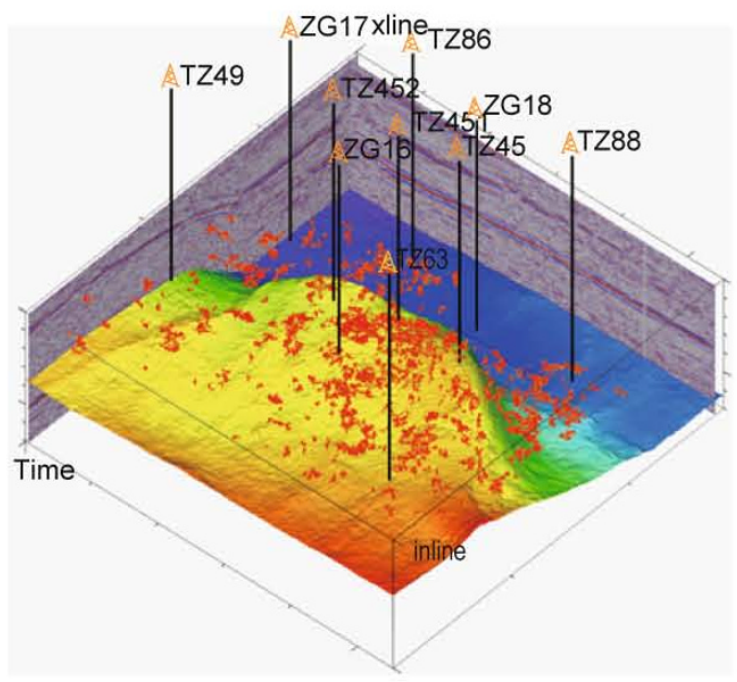

Fig. 113 -D characterization volume of the Ordovician carbonate reservoirs

\section{Conclusions}

(1) The multi-attribute optimization method, which is formed on the basis of statistics, integrates various seismic attributes and eliminates non-useful seismic attributes by multivariate step-by-step discrimination. Only those attributes which are more effective are preserved in the final discriminant. On the other hand, by employing the kernel principal component analysis method, repetition and redundancy in seismic data is avoided while preserving the information in the raw data. The stability is ensured and the reliability is improved by dealing with the non-linear relationships among various attributes.

(2) Considering the characteristics of carbonate reservoirs in the Tarim Basin, a series of 3-D seismic attributes including coherence analysis, spectra decomposition using non-orthogonal Gabor-Morlet wavelet transform, seismic absorption attenuation analysis and well-constrained impedance inversion is formed by an integrated analysis of seismic, geology and logging data in the Tazhong 45 area. Moreover, those attributes are optimized using multi-attribute discriminant analysis and kernel principle component analysis, further improving the capacity of attributes in predicting the spatial distribution of carbonate reservoirs. It is significant to the discovery and production of carbonate reservoirs in the Tarim Basin and other areas with similar geological settings.

\section{Acknowledgements}

The work is co-supported by the National Basic Research Program of China (Grant No.2011CB201103) and the National Science and Technology Major Project (Grant 
No.2011ZX05004003). The authors would like to thank Lab for Integration of Geology and Geophysics (LIGG) at China University of Petroleum for the permission to publish this work and Tarim Oilfield Co., PetroChina for their help in providing field data.

\section{References}

Bi Y B, Long S X, Guo T L, et al. Prediction of gas distribution with frequency attenuation attribute in TNB area. Oil \& Gas Geology. 2007. 28(1): 116-120 (in Chinese)

Cai R. Carbonate cave identification by using a spectral decomposition technique. Petroleum Exploration and Development. 2005. 32(2): 8285 (in Chinese)

Chen G P, Pan J G and Tao Y G. The application of multiple information for prediction of carbonate karst reservoir and effect analysis. Geophysical Prospecting for Petroleum. 2005. 44(1): 33-36 (in Chinese)

Han G H, Qi L X, Li Z J, et al. Prediction of the Ordovician fracturedvuggy carbonate reservoirs in Tahe oilfield. Oil \& Gas Geology. 2006. 27(6): 860-870 (in Chinese)

Huang Z Y, Wang Y J and Su Y C. A new analysis method for seismic wave attenuation: an effective tool for predicting hydrocarbon anomaly. Oil Geophysical Prospecting. 2000. 35(6): 768-773 (in Chinese)

Jin J, Tan J Q, Li T M, et al. Reservoir sands description using well log constrain inversion method. Xinjiang Petroleum Geology. 2003. 24(1): $72-74$ (in Chinese)

Liu L, Xing Z Y, Sun G, et al. The application of well log constrained seismic inversion to the description of reservoirs in Zhuang-106 block. Petroleum Exploration and Development. 1999. 26(5): 80-82 (in Chinese)

Pang X Q, Tian J, Pang H, et al. Main progress and problems in research on Ordovician hydrocarbon accumulation in the Tarim Basin. Petroleum Science. 2010. 7(2): 147-163

Sams M S, Neep J P, Worthington M H, et al. The measurement of velocity dispersion and frequency-dependent intrinsic attenuation in sedimentary rocks. Geophysics. 1997. 62(5): 1456-1464

Sinha S, Routh P S, Anno P D, et al. Spectral decomposition of seismic data with continuous-wavelet transform. Geophysics. 2005. 70(6): 19-25

Sun Z D. Seismic methods for heavy oil reservoir monitoring and characterization. Ph.D. Thesis. Calgary: University of Calgary, Department of Geology and Geophysics. 1999. 108-117

Wang S X, Cao H L, Jin W F, et al. Seismic response and prediction of fracture-cavity system in carbonate reservoir: A case study in the Ka-1 field. Geophysical Prospecting for Petroleum. 2005. 44(5): 421-427 (in Chinese)

Wang W L, Pang X Q, Liu L F, et al. Ordovician carbonate reservoir bed characteristics and reservoir-forming conditions in the Lungudong region of the Tarim Basin. Acta Geologica Sinica. 2010. 84(5): 1170-1179

Wen Z X, Wang H M, Qi L X, et al. Research on the prediction of Ordovician carbonate reservoirs of fissure-cave type in the Tahe oilfield. Earth Science Frontiers. 2008.15(1): 94-100 (in Chinese)

Yin X Y and Zhou J Y. Summary of optimum methods of seismic attributes. Oil Geophysical Prospecting. 2005. 40(4): 482-489 (in Chinese)

Yuan S J. Application of spectrum decomposition of nonorthogonal wavelet transforms to reservoir prediction in the Daniudi gas field. Natural Gas Industry. 2007. 27(12): 52-54 (in Chinese)

(Edited by Hao Jie) 